\title{
一种荧光增强型三价金属离子荧光探针的合成及性质
}

\author{
侯淑华王思琦曲忠国钟克利边延江汤立军*
}

(渤海大学化学化工学院 锦州 121013)

\begin{abstract}
摘要 设计合成一种新型罗丹明类探针 $\mathbf{L}$, 并研究了其光谱性质. 实验结果表明, $\mathbf{L}$ 在甲醇/水 $[V: V=8: 2, c($ Tris $)=10$ $\mathrm{mmol} / \mathrm{L}, \mathrm{pH}=7.2]$ 体系中对三价金属离子 $\left(\mathrm{Cr}^{3+} 、 \mathrm{Fe}^{3+} 、 \mathrm{Al}^{3+}\right)$ 具有苂光增强性响应, 很好的选择性和较高的灵敏度, 不受 其它二价及一价金属离子的影响，抗干扰能力强. 探针 $\mathbf{L}$ 对 $\mathrm{Cr}^{3+} 、 \mathrm{Fe}^{3+} 、 \mathrm{Al}^{3+}$ 的紫荧光检测限分别为 $2.1 \times 10^{-4} 、 2.3 \times$ $10^{-4}$ 和 $4.4 \times 10^{-4} \mathrm{~mol} / \mathrm{L}$, 表明其在各种水体样品中对三价金属离子的检测具有潜在的应用价值.

关键词 罗丹明; 三价金属离子; 荧光增强
\end{abstract}

\section{A Fluorescence Enhancement Probe for Trivalent lons: Preparation and Property}

\author{
Hou, Shuhua $\quad$ Wang, Siqi $\quad$ Qu, Zhongguo Zhong, Keli $\quad$ Bian, Yanjiang $\quad$ Tang, Lijun* \\ (Department of Chemistry, Bohai University, Jinzhou 121013)
}

\begin{abstract}
A novel rhodamine-based probe $\mathbf{L}$ was synthesized and characterized. The results showed that probe $\mathbf{L}$ acted as a fluorescent enhancement probe for $\mathrm{Cr}^{3+}, \mathrm{Fe}^{3+}$ and $\mathrm{Al}^{3+}$ recognition in $\mathrm{CH}_{3} \mathrm{OH} / \mathrm{H}_{2} \mathrm{O}(V: V=8: 2, c$ (Tris) $=10 \mathrm{mmol} / \mathrm{L}$, $\mathrm{pH}=7.2)$. $\mathbf{L}$ is highly selective to trivalent ions $\mathrm{Cr}^{3+}, \mathrm{Fe}^{3+}$ and $\mathrm{Al}^{3+}$ over other monovalent or divalent ions. Detection limits of probe $\mathbf{L}$ for $\mathrm{Cr}^{3+}, \mathrm{Fe}^{3+}$ and $\mathrm{Al}^{3+}$ are $2.1 \times 10^{-4}, 2.3 \times 10^{-4}$ and $4.4 \times 10^{-4} \mathrm{~mol} / \mathrm{L}$, respectively, which make it have potentially application value in $\mathrm{Cr}^{3+}, \mathrm{Fe}^{3+}$ and $\mathrm{Al}^{3+}$ detection in various water samples.
\end{abstract}

Keywords rhodamine; trivalent ions; fluorescence enhancement

三价金属离子尤其是 $\mathrm{Cr}^{3+} 、 \mathrm{Fe}^{3+}$ 和 $\mathrm{Al}^{3+}$ 具有非常重 要的生物学功能. 细胞内 $\mathrm{Cr}^{3+} 、 \mathrm{Fe}^{3+} 、 \mathrm{Al}^{3+}$ 的浓度直接 影响细胞功能, 进而影响整个代谢, 与人类健康息息相 关. 如人体内 $\mathrm{Cr}^{3+}$ 的失衡会引发糖尿病和动脉硬化等多 种疾病 ${ }^{[1]}$, 参与人体细胞新陈代谢的 $\mathrm{Fe}^{3+}$ 的失衡会导致 贫血、肝损伤、肾衰竭等 ${ }^{[2]}$. 虽然 $\mathrm{Al}^{3+}$ 不属于人体所必 需的微量元素, 但平时过多摄入 $\mathrm{Al}^{3+}$ 会引发老年痴呆 ${ }^{[3]}$. 因此, 快速检测三价金属离子势在必行.

目前化学和生物领域通常采用 $\mathrm{X}$ 射线光电子能谱 (XPS)、原子光谱法、质谱法、电感耦合等离子发射光 谱(ICP)等方法检测金属离子 ${ }^{[4]}$. 以上方法具有高效、迅 速和精确等优点, 但是由于需要大型设备和复杂操作, 特别是在生物体内对相关离子和三价离子的识别, 具有 很大的局限性. 苂光分子探针因具有灵敏度高、选择性 好、响应时间短、可直接观察等优点而被广泛应用于各
种金属离子的检测. 由于 $\mathrm{Fe}^{3+}$ 和 $\mathrm{Cr}^{3+}$ 的顺磁性，使得很 多荧光探针与 $\mathrm{Fe}^{3+}$ 和 $\mathrm{Cr}^{3+}$ 作用后, 表现为苂光淬灭, 严 重影响检测的灵敏度 ${ }^{[5]}$. 罗丹明类染料由于其易与金属 离子作用发生开环反应，进而表现为荧光增强，已经被 广泛应用于分子探针设计. 目前, 罗丹明类荧光探针多 用于检测一种金属离子 $\mathrm{Ag}^{+} 、 \mathrm{Zn}^{2+} 、 \mathrm{Cu}^{2+} 、 \mathrm{Hg}^{2+} 、 \mathrm{Fe}^{2+}$ 、 $\mathrm{Fe}^{3+} 、 \mathrm{Cr}^{3+}$ 和 $\mathrm{Al}^{3+}$ 等, 作用和功能单一 ${ }^{[6]}$; 检测 $\mathrm{Fe}^{3+}$ 、 $\mathrm{Cr}^{3+}$ 和 $\mathrm{Al}^{3+}$ 需要采用不同的苂光探针, 增加了检测成本 和检测时间. 目前能够同时检测 $\mathrm{Fe}^{3+} 、 \mathrm{Cr}^{3+}$ 和 $\mathrm{Al}^{3+}$ 高灵 敏度、强抗干扰能力的荧光探针较少 ${ }^{[7]}$. 前期我们通过 “点击化学” 合成了苯并噻唑-罗丹明衍生物, 在甲醇溶 液中对三价金属离子 $\mathrm{Fe}^{3+} 、 \mathrm{Cr}^{3+}$ 和 $\mathrm{Al}^{3+}$ 具有很好的选择 性 ${ }^{[8]}$. 然而，在有机溶剂中检测金属离子限制了其广泛 应用. 因此, 本文拟增加罗丹明苂光探针的亲水性, 在 罗丹明 $\mathrm{B}$ 上引入亲水基团醚链，期望增强其亲水性的同

\footnotetext{
* Corresponding author. E-mail: bhutang@126.com

Received December 2, 2016; revised January 24, 2017; published online February 15, 2017.

Project supported by the Program for Liaoning Excellent Talents in University (No. LR2015001).

辽宁省高等学校优秀人才支持计划(No. LR2015001)资助项目.
} 
时对金属离子还有一定的识别效果(Eq. 1).

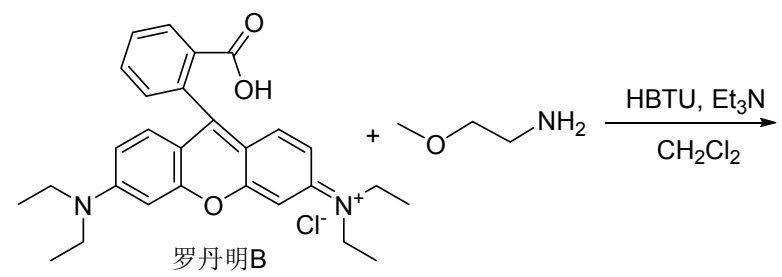<smiles>CCN(CC)c1ccc2c(c1)Oc1cc(N(CC)CC)ccc1C21c2ccccc2C(=O)N1CCOC</smiles>

\section{1 结果与讨论}

\section{1 荧光探针 L 对金属离子的选择性识别}

为了研究化合物 $\mathbf{L}$ 对金属离子的识别性能, 选择 $\mathrm{Zn}^{2+} 、 \mathrm{~Pb}^{2+} 、 \mathrm{Cu}^{2+} 、 \mathrm{Fe}^{2+} 、 \mathrm{Fe}^{3+} 、 \mathrm{Cd}^{2+} 、 \mathrm{Mn}^{2+} 、 \mathrm{Co}^{2+} 、$ $\mathrm{Ni}^{+} 、 \mathrm{Cr}^{3+} 、 \mathrm{Al}^{3+} 、 \mathrm{Ag}^{+} 、 \mathrm{Na}^{+} 、 \mathrm{Ba}^{2+} 、 \mathrm{Sr}^{2+} 、 \mathrm{Hg}^{2+} 、 \mathrm{~K}^{+} 、$ $\mathrm{Mg}^{2+}$ 为研对象, 发现探针在甲醇/水 $[V: V=8: 2$, $c$ (Tris) $=10 \mathrm{mmol} / \mathrm{L}, \mathrm{pH}=7.2]$ 体系中, 对 $\mathrm{M}^{3+}\left(\mathrm{Fe}^{3+}\right.$ 、 $\mathrm{Cr}^{3+} 、 \mathrm{Al}^{3+}$ )具有良好的选择性，溶液颜色在加入 $\mathrm{M}^{3+}$ $\left(\mathrm{Fe}^{3+} 、 \mathrm{Cr}^{3+} 、 \mathrm{Al}^{3+}\right)$ 后由无色变为粉红色. 测定紫外光谱, 我们发现化合物 $\mathbf{L}$ 溶液中加入 $\mathrm{M}^{3+}\left(\mathrm{Fe}^{3+} 、 \mathrm{Cr}^{3+} 、 \mathrm{Al}^{3+}\right)$ 后, 吸收峰在 $565 \mathrm{~nm}$ 处明显增强, 而化合物 $\mathbf{L}$ 本身及加 入其它金属离子在 $565 \mathrm{~nm}$ 处无吸收峰出现(图 1A). 随 后我们也进行了苂光光谱的测试(图 1B), 化合物 $\mathbf{L}$ 本身 和其他金属阳离子并没有引发任何的荧光变化, 但在 $\mathrm{M}^{3+}\left(\mathrm{Fe}^{3+} 、 \mathrm{Cr}^{3+} 、 \mathrm{Al}^{3+}\right)$ 存在时, 在 $590 \mathrm{~nm}$ 处产生发射 峰, 并伴随着强烈的橙色荧光. 因此, 化合物 $\mathbf{L}$ 是对 $\mathrm{M}^{3+}\left(\mathrm{Fe}^{3+} 、 \mathrm{Cr}^{3+} 、 \mathrm{Al}^{3+}\right)$ 具有高选择性的荧光增强型探 针.

\section{2 共存离子的影响}

在化合物 $\mathbf{L}$ 与共存金属离子溶液中加入相同浓度 倍量的 $\mathrm{Cr}^{3+}$, 混合均匀后, 测试苂光变化. 如图 2 所示, 所有被加入的金属阳离子对其苂光发射强度几乎没有 影响, 即化合物 $\mathbf{L}$ 与 $\mathrm{Cr}^{3+}$ 作用的苂光强度几乎不受这些 共存离子的干扰. 同样, 对于 $\mathrm{Fe}^{3+} 、 \mathrm{Al}^{3+}$, 化合物 $\mathbf{L}$ 与 $\mathrm{Fe}^{3+} 、 \mathrm{Al}^{3+}$ 作用的苂光强度几乎不受这些共存离子的干 扰. 说明化合物 $\mathbf{L}$ 对三价金属离子 $\mathrm{Fe}^{3+} 、 \mathrm{Al}^{3+} 、 \mathrm{Cr}^{3+}$ 识 别具有较强的抗干扰能力.

\section{3 不同浓度的 $\mathrm{Cr}^{3+} 、 \mathrm{Fe}^{3+} 、 \mathrm{Al}^{3+}$ 对化合物 $\mathrm{L}$ 光谱的 影响}

在甲醇/水 $[V: V=8: 2, c$ (Tris) $=10 \mathrm{mmol} / \mathrm{L}, \mathrm{pH}=$
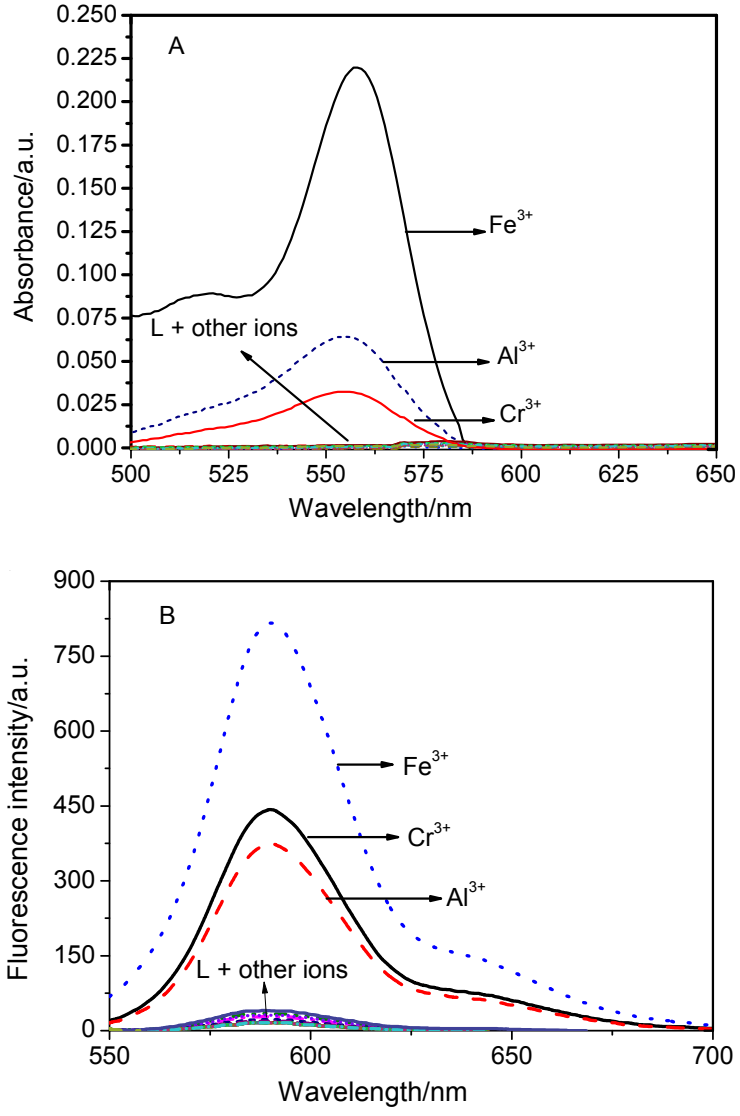

图 1 加入不同金属离子化合物 $\mathbf{L}(10 \mu \mathrm{mol} / \mathrm{L})$ 的紫外光谱(A) 和荧光光谱 $(B)\left(\lambda_{\mathrm{ex}}=530 \mathrm{~nm}\right)$

Figure 1 Absorbance (A) and fluorescence spectra (B) $\left(\lambda_{\mathrm{ex}}=\right.$ $530 \mathrm{~nm})$ of $\mathbf{L}(10 \mu \mathrm{mol} / \mathrm{L})$ upon addition of different metal ions

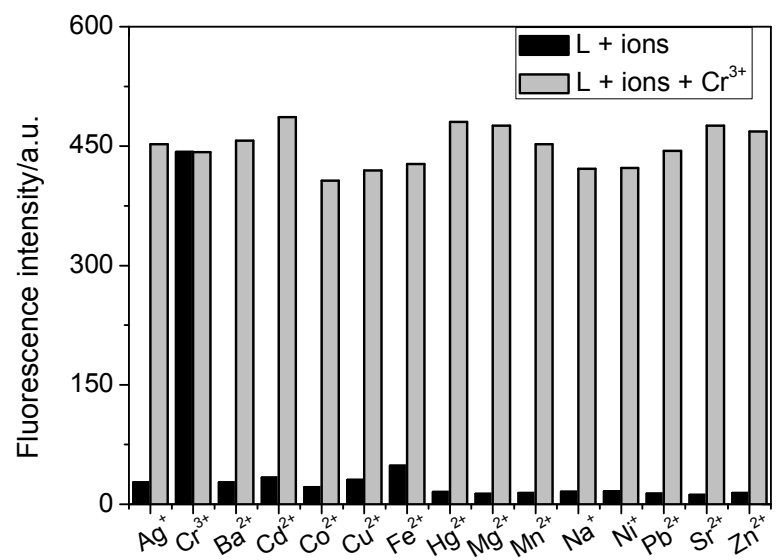

图 2 其它金属离子和 $\mathrm{Cr}^{3+}$ 共存时探针 $\mathbf{L}(10 \mu \mathrm{mol} / \mathrm{L})$ 在 $\mathrm{CH}_{3} \mathrm{OH} / \mathrm{H}_{2} \mathrm{O}$ 中荧光发射光谱的变化 $\left(\lambda_{\mathrm{ex}}=530 \mathrm{~nm}\right)$

Figure 2 Fluorescence emission spectrum of probe $\mathbf{L}$ (10 $\mu \mathrm{mol} / \mathrm{L})$ to $\mathrm{Cr}^{3+}$ upon addition of various metal ions in $\mathrm{CH}_{3} \mathrm{OH} /$ $\mathrm{H}_{2} \mathrm{O}\left(\lambda_{\mathrm{ex}}=530 \mathrm{~nm}\right)$

7.2]溶液中, 测定了不同 $\mathrm{Cr}^{3+} 、 \mathrm{Fe}^{3+} 、 \mathrm{Al}^{3+}$ 浓度条件下化 合物 $\mathbf{L}$ 溶液的紫外吸收光谱和苂光发射光谱. 当 $\mathrm{Cr}^{3+}$ 的 浓度不断增加, 其最大紫外吸收(550 nm) 和最大苂光发 
射(590 nm)强度不断增强. $\mathrm{Fe}^{3+}$ 和 $\mathrm{Al}^{3+}$ 也有类似的变化 规律, 当 $\mathrm{Cr}^{3+}$ 浓度为探针 $\mathbf{L}$ 浓度的 80 equiv. 时, 紫外吸 收和苂光强度达到最大值, 证明其达到饱和(图 3). 实验 发现, 化合物 $\mathbf{L}$ 的紫外吸收和苂光强度与 $\mathrm{Cr}^{3+}$ 浓度有较 好的线性关系，表明化合物 $\mathbf{L}$ 可以用于定量检测 $\mathrm{Cr}^{3+}$ 浓 度. 同样的, 随着 $\mathrm{Al}^{3+} 、 \mathrm{Cr}^{3+}$ 浓度的增加, 化合物 $\mathbf{L}$ 最 大紫外吸收(550 nm) 和最大荧光发射 $(590 \mathrm{~nm})$ 强度逐渐 增强, 当 $\mathrm{Fe}^{3+}$ 浓度为化合物 $\mathbf{L}$ 浓度的 100 倍、 $\mathrm{Al}^{3+}$ 浓度 为化合物 $\mathbf{L}$ 浓度的 110 倍时达到最高值. 其中化合物 $\mathbf{L}$ 对 $\mathrm{Cr}^{3+} 、 \mathrm{Fe}^{3+} 、 \mathrm{Al}^{3+}$ 的紫外检测限分别为 $1.4 \times 10^{-4}$ 、 $9.5 \times 10^{-5}$ 和 $3.6 \times 10^{-4} \mathrm{~mol} / \mathrm{L}$; 苂光检测限分别为 $2.1 \times$ $10^{-4} 、 2.3 \times 10^{-4}$ 和 $4.4 \times 10^{-4} \mathrm{~mol} / \mathrm{L}$.
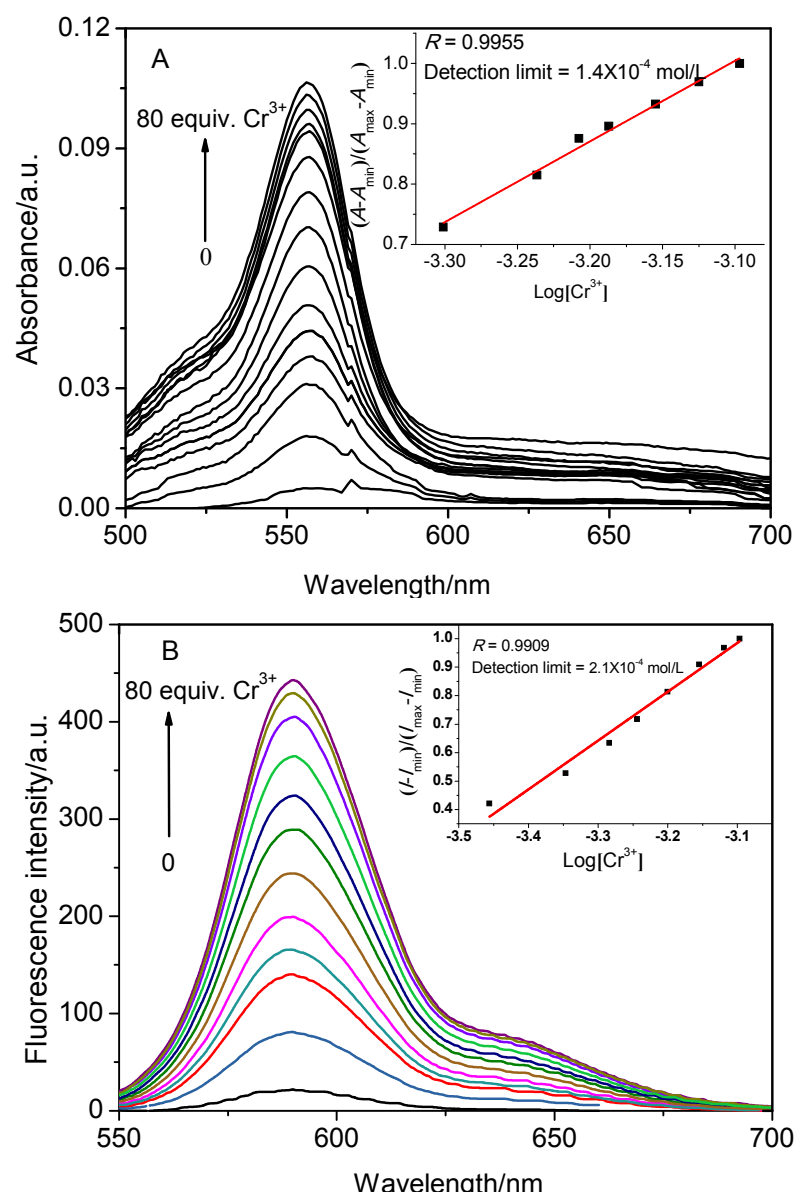

图 $3 \mathbf{L}(10 \mu \mathrm{mol} / \mathrm{L})$ 中不同浓度 $\mathrm{Cr}^{3+}$ 的紫外光谱(A)和苂光发 射光谱 $(B)$ 的变化曲线图 $\left(\lambda_{\mathrm{ex}}=530 \mathrm{~nm}\right)$

Figure 3 Absorbance spectrum (A) and fluorescence emission spectrum (B) of probe $\mathbf{L}$ in the presence of $\mathrm{Cr}^{3+}$ with different concentration $\left(\lambda_{\mathrm{ex}}=530 \mathrm{~nm}\right)$

$1.4 \mathrm{pH}$ 对化合物 $\mathrm{L}$ 和 $\mathrm{L}+\mathrm{M}^{3+}\left(\mathrm{Cr}^{3+} 、 \mathrm{Fe}^{3+} 、 \mathrm{Al}^{3+}\right)$ 荧光 光谱的影响

为了考察溶液的 $\mathrm{pH}$ 对化合物 $\mathbf{L}$ 的荧光响应的影响, 实验测定了不同 $\mathrm{pH}$ 值的 $V\left(\mathrm{CH}_{3} \mathrm{OH}\right): V($ Tris- $\mathrm{HCl})=1$ : 1 溶液的苂光强度. 图 4 为化合物 $\mathbf{L}$ 及 $\mathbf{L}+\mathbf{M}^{3+}$ 分别在其
最大发射波长 $(590 \mathrm{~nm})$ 处的苂光强度与 $\mathrm{pH}$ 关系曲线. 可以看出, $\mathrm{pH}$ 为 $2 \sim 8$ 之间, $\mathbf{L}$ 几乎没有苂光, 当加入了 三价金属离子之后，在 $\mathrm{pH}$ 为 3 7 之间， $\mathbf{L}$ 的苂光强度 大幅度增强. 由此可见, 探针 $\mathbf{L}$ 在弱酸性和中性条件下 可以实现对三价金属离子的检测.

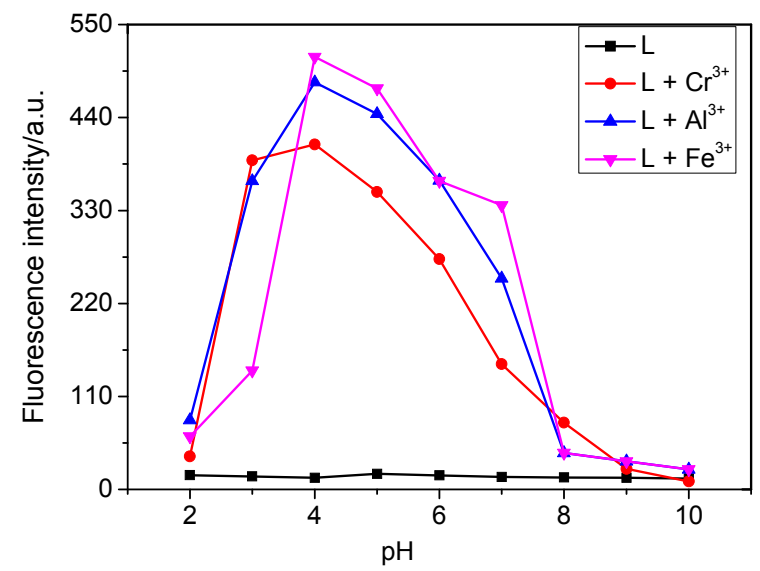

图 4 在三价金属离子存在或不存在的条件下，探针 $\mathbf{L}(10$ $\mu \mathrm{mol} / \mathrm{L})$ 在不同 $\mathrm{pH}$ 值时荧光强度的变化 $\left(\lambda_{\mathrm{ex}}=530 \mathrm{~nm}\right)$

Figure 4 Change in fluorescence intensity of probe $\mathbf{L}(10$ $\mu \mathrm{mol} / \mathrm{L})$ measured in the absence and presence of trivalent metals $\left(\lambda_{\mathrm{ex}}=530 \mathrm{~nm}\right)$

\section{5 探针 $\mathrm{L}$ 试纸对 $\mathrm{Cr}^{3+} 、 \mathrm{Fe}^{3+} 、 \mathrm{Al}^{3+}$ 的识别}

将滤纸浸泡至 $\mathbf{L}(1 \mathrm{mmol} / \mathrm{L})$ 甲醇溶液中, 完全浸透 后取出晾干. 滴加 $\mathrm{Cr}^{3+} 、 \mathrm{Fe}^{3+} 、 \mathrm{Al}^{3+}$ 溶液至制备的试纸 上，在紫外灯(365 nm)照射下，未滴加金属离子及与未 浸泡 $\mathbf{L}$ 的空白滤纸均发出蓝色光, 为滤纸增白剂发出的 光; 滴加三价金属离子的发出桔色荧光(图 5), 表明探 针 $\mathbf{L}$ 可以制备成试纸用以检测三价金属离子.

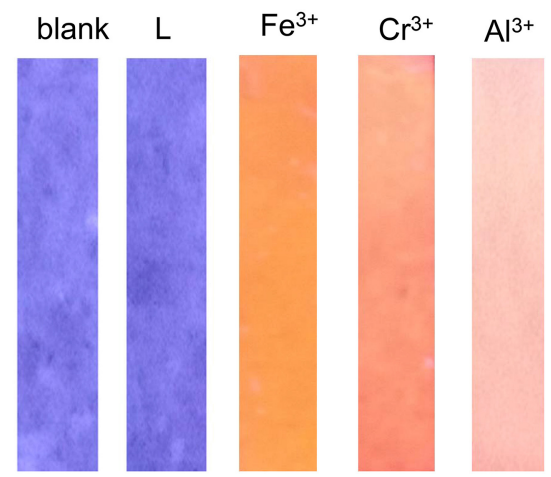

图 5 检测三价金属离子试纸

Figure 5 Paper test strips for trivalent ions detection

\section{6 探针 $\mathrm{L}$ 和 $\mathrm{Cr}^{3+} 、 \mathrm{Fe}^{3+} 、 \mathrm{Al}^{3+}$ 的络合方式}

为了探索三价金属离子 $\mathrm{Cr}^{3+} 、 \mathrm{Fe}^{3+} 、 \mathrm{Al}^{3+}$ 与探针 $\mathbf{L}$ 之间的作用机理，采用等物质的量的连续变化法(Job's Plot)计算两者的络合比. 随着 $\mathrm{Cr}^{3+}$ 摩尔分数的不断改 
变, 体系的荧光强度在 $590 \mathrm{~nm}$ 处随之变化(图 6). 当 $\mathrm{Cr}^{3+}$ 摩尔分数为 0.5 时, 探针 $\mathbf{L}$ 与 $\mathrm{Fe}^{3+}$ 形成的络合物在 $590 \mathrm{~nm}$ 处出现最大的苂光强度, 说明探针 $\mathbf{L}$ 与 $\mathrm{Cr}^{3+}$ 的结 合比为 $1: 1$. 同样的, $\mathrm{Fe}^{3+}$ 和 $\mathrm{Cr}^{3+}$ 也有类似的结果, 结 合比为 $1: 1$. 为了探讨三价金属离子与探针的结合方 式, 我们测试了探针 $\mathbf{L}$ 及加入 $\mathrm{Al}^{3+}$ (4 equiv.)的氢谱图 (图 7), 发现加入探针 $\mathbf{L}$ 后 $\mathrm{a}$ 和 $\mathrm{b}$ 氢的化学位移均向低场 移动 $\delta 0.08$, 说明内酰胺的 $\mathrm{N}$ 和梄链中的 $\mathrm{O}$ 均参与 $\mathbf{L}$ 与 三价金属离子的螯合; $\mathrm{c}$ 氢的化学位移向高场移动 $\delta$ 0.06 , 可能是由于螯合金属离子后内酰胺开环甲氧基中 甲基移动至罗丹明结构中共轭体系的上方产生屏蔽作 用. 推测其络合机理可能如 Eq. 2 所示. 探针 $\mathbf{L}$ 以内酰胺 的结构稳定存在无荧光, 加入三价金属离子 $\mathrm{Fe}^{3+} 、 \mathrm{Al}^{3+}$ 、 $\mathrm{Cr}^{3+}$ 后内酰胺开环发出橙色苂光.

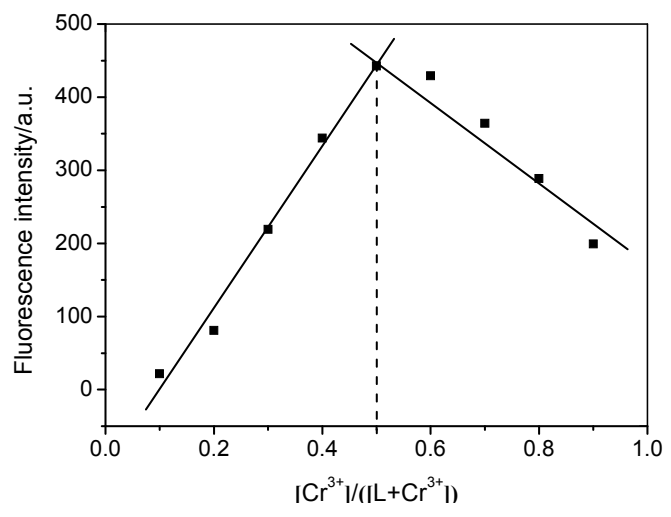

图 6 探针 $\mathbf{L}$ 和 $\mathrm{Cr}^{3+}$ 的 Job 曲线 $\left(\lambda_{\mathrm{ex}}=530 \mathrm{~nm}\right)$

Figure 6 Job's plot of probe $\mathrm{L}$ and $\mathrm{Cr}^{3+}\left(\lambda_{\mathrm{ex}}=530 \mathrm{~nm}\right)$

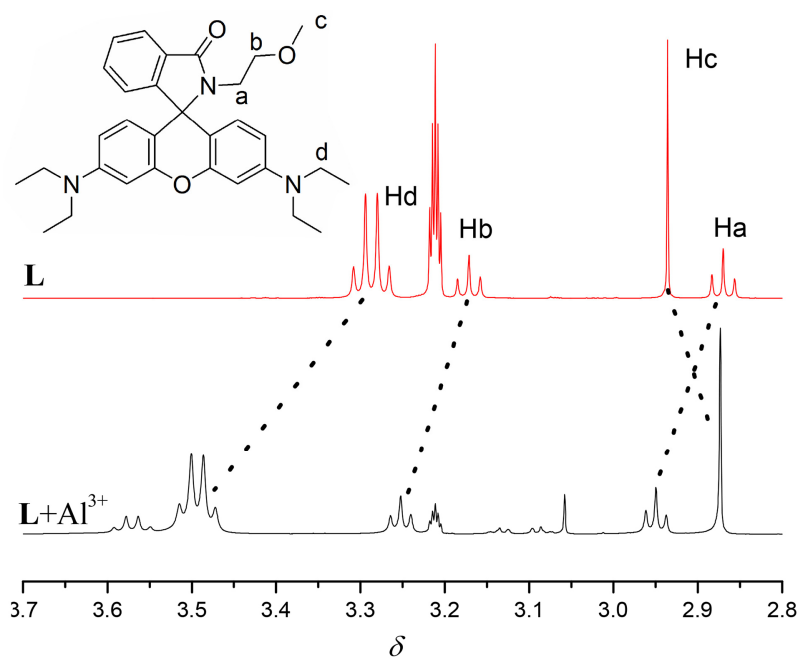

图 7 探针 $\mathbf{L}$ 及 $\mathbf{L}+\mathrm{Al}^{3+}$ 在気代甲醇中部分 ${ }^{1} \mathrm{H} \mathrm{NMR}$ 图 Figure 7 Partial ${ }^{1} \mathrm{H}$ NMR spectra of $\mathbf{L}$ and $\mathbf{L}+\mathrm{Al}^{3+}$ measured in $\mathrm{CD}_{3} \mathrm{OD}$

\section{2 结论}

本文通过一步法成功将亲水基团醚链引入至罗丹

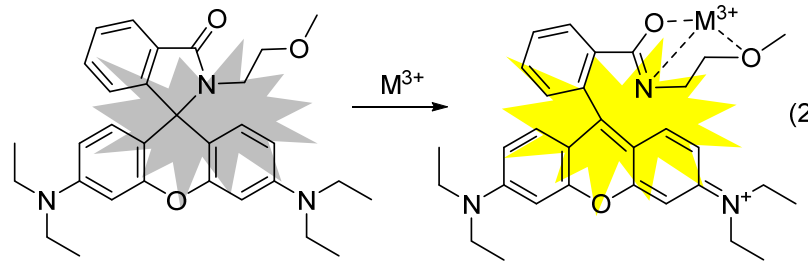

(2)

明 B 结构上得到了一种苂光增强型探针 $\mathbf{L}$, 并对其结构 进行了表征. 测定了 $\mathbf{L}$ 在甲醇/水 $[V: V=8: 2, c($ Tris $)=$ $10 \mathrm{mmol} / \mathrm{L}, \mathrm{pH}=7.2]$ 体系中的紫外和荧光光谱. 研究发 现苂光探针 $\mathbf{L}$ 对三价金属离子 $\left(\mathrm{Cr}^{3+} 、 \mathrm{Fe}^{3+} 、 \mathrm{Al}^{3+}\right)$ 具有很 好的选择性和较高的灵敏度, 不受其它二价金属离子和 一价金属离子的影响. $\mathbf{L}$ 对 $\mathrm{Cr}^{3+} 、 \mathrm{Fe}^{3+} 、 \mathrm{Al}^{3+}$ 的紫外检测 限分别为 $1.4 \times 10^{-4} 、 9.5 \times 10^{-5}$ 和 $3.6 \times 10^{-4} \mathrm{~mol} / \mathrm{L}$; 苂 光检测限分别为 $2.1 \times 10^{-4} 、 2.3 \times 10^{-4}$ 和 $4.4 \times 10^{-4}$ $\mathrm{mol} / \mathrm{L}$.

\section{3 实验部分}

\section{1 仪器与试剂}

500 NMR 核磁共振仪(德国 Bruker 公司); MALDI-TOF MS 基质辅助飞行时间质谱仪(Bruker attoflex III smartbeam, Germany); 苂光分光光度计(970CRT, 上海 三科).

苯并三氮唑 $-N, N, N^{\prime}, N^{\prime}$ - 四甲基腿六氟磷酸酯 (HBTU)购自阿拉丁, 其它试剂均为国产市售分析纯, 所有溶剂均经干燥处理. 金属离子溶液用相应的盐溶于 纯净水制得.

\section{2 化合物 $L$ 的合成}

罗丹明 B (479 mg, $1.0 \mathrm{mmol}$ )和 2-甲氧基乙胺(94 $\mathrm{mg}, 1.3 \mathrm{mmol}$ ) 溶解于干燥的二氯甲烷中, 加入苯并三氮 唑- $N, N, N^{\prime}, N^{\prime}$-四甲基艮六氟磷酸酯(HBTU) (568 mg, 1.5 $\mathrm{mmol}$ )和三乙胺 $(152 \mathrm{mg}, 1.5 \mathrm{mmol})$. 在室温下过夜反应, 薄层色谱(TLC)跟踪, 反应完全后, 浓缩柱层析得到白 色固体 $349 \mathrm{mg}$, 收率为 70.5\%. m.p. 268.7 269.5 ${ }^{\circ} \mathrm{C} ;{ }^{1} \mathrm{H}$ NMR $\left(500 \mathrm{MHz}, \mathrm{CDCl}_{3}\right) \delta: 7.92(\mathrm{dd}, J=6.8,2.9 \mathrm{~Hz}, 1 \mathrm{H})$, $7.44(\mathrm{dd}, \quad J=6.8,2.9 \mathrm{~Hz}, 2 \mathrm{H}), 7.10(\mathrm{dd}, J=6.8,3.2 \mathrm{~Hz}$, 1H), $6.48(\mathrm{~s}, 1 \mathrm{H}), 6.46(\mathrm{~s}, 1 \mathrm{H}), 6.40(\mathrm{br}, 2 \mathrm{H}), 6.29(\mathrm{br}, 2 \mathrm{H})$, $3.38 \sim 3.33(\mathrm{~m}, 10 \mathrm{H}), 3.12(\mathrm{~s}, 3 \mathrm{H}), 3.07(\mathrm{t}, J=6.9 \mathrm{~Hz}, 2 \mathrm{H})$, $1.18(\mathrm{t}, J=7.0 \mathrm{~Hz}, 12 \mathrm{H}) ;{ }^{13} \mathrm{C}$ NMR $\left(100 \mathrm{MHz}, \mathrm{CDCl}_{3}\right) \delta$ : $168.3,153.7,153.2,148.7,132.3,131.0,128.9,127.9$, 123.7, 122.8, 108.0, 105.6, 97.7, 69.3, 64.7, 58.4, 44.3, 39.1, 12.5. HRMS $\left(\mathrm{ESI}^{+}\right)$calcd for $\mathrm{C}_{31} \mathrm{H}_{38} \mathrm{~N}_{3} \mathrm{O}_{3}[\mathrm{M}+\mathrm{H}]^{+}$: 500.2908, found 500.2920.

\section{3 光谱测定方法}

化合物 $\mathbf{L}$ 以二甲基亚砜(DMSO)为溶剂配制成 
$1.0 \times 10^{-3} \mathrm{~mol} / \mathrm{L}$ 的溶液, 然后用甲醇稀释成 $1.0 \times 10^{-5}$ $\mathrm{mol} / \mathrm{L}$ 溶液; 以二次蒸馏水为溶剂, 配制 $5 \times 10^{-3} \mathrm{~mol} / \mathrm{L}$ 的离子溶液 $\left(\mathrm{ZnCl}_{2} 、 \mathrm{~Pb}\left(\mathrm{NO}_{3}\right)_{2} 、 \mathrm{Cu}\left(\mathrm{NO}_{3}\right)_{2} \bullet 3 \mathrm{H}_{2} \mathrm{O} 、 \mathrm{FeCl}_{2} \bullet\right.$ $4 \mathrm{H}_{2} \mathrm{O} 、 \mathrm{NaCl} 、 \mathrm{Fe}\left(\mathrm{NO}_{3}\right)_{3} \bullet 9 \mathrm{H}_{2} \mathrm{O} 、 \mathrm{Cd}\left(\mathrm{NO}_{3}\right)_{2} \bullet 4 \mathrm{H}_{2} \mathrm{O} 、 \mathrm{MnCl}_{2}$ 、 $\mathrm{Co}\left(\mathrm{NO}_{3}\right)_{2} \cdot 6 \mathrm{H}_{2} \mathrm{O} 、 \mathrm{Ni}\left(\mathrm{NO}_{3}\right)_{2} \cdot 6 \mathrm{H}_{2} \mathrm{O} 、 \mathrm{Cr}\left(\mathrm{NO}_{3}\right)_{3} \bullet 9 \mathrm{H}_{2} \mathrm{O}$ 、 $\mathrm{Al}\left(\mathrm{NO}_{3}\right)_{3} \bullet 9 \mathrm{H}_{2} \mathrm{O} 、 \mathrm{AgClO}_{4} 、 \mathrm{Ba}\left(\mathrm{NO}_{3}\right)_{2} 、 \mathrm{Sr}\left(\mathrm{NO}_{3}\right)_{2} 、 \mathrm{HgCl}_{2}$ 、 $\mathrm{KNO}_{3} 、 \mathrm{MgCl}_{2}$ ), 以留备用. 测试条件: 室温, 样品池为 1 $\mathrm{cm} \times 1 \mathrm{~cm} \times 4 \mathrm{~cm}$ 石英比色典, 灵敏度 3 , 激发狭缝宽 5 $\mathrm{nm}$, 发射狭缝宽 $10 \mathrm{~nm}$, 激发波长 $\lambda_{\mathrm{ex}}$ 为 $530 \mathrm{~nm}$, 于 $550 \sim$ $750 \mathrm{~nm}$ 范围内检测荧光.

辅助材料 (Supporting Information) 探针 $\mathbf{L}$ 的 ${ }^{1} \mathrm{H}$ $\mathrm{NMR} 、{ }^{13} \mathrm{C} \mathrm{NMR}$ 和高分辨质谱图，以及探针 $\mathbf{L}$ 对 $\mathrm{Fe}^{3+}$ 、 $\mathrm{Al}^{3+}$ 识别性能. 这些材料可以免费从本刊网站 (http://sioc-journal.cn)上下载.

\section{References}

[1] (a) Singh, A. K.; Gupta, V.; Gupta, B. Anal. Chim. Acta 2007, 585, 171.

(b) Vincent J B. Nutr. Rev. 2000, 58(3):67.

[2] (a) Pithadia, A. S.; Lim, M. H. Curr. Opin. Chem. Biol. 2012, 16, 67.

(b) Weizman, H.; Ardon, O.; Mester, B.; Libman, J.; Dwir, O.; Hadar, Y.; Chen, Y.; Shanzer, A. J. Am. Chem. Soc. 1996, 118, 12368.

(c) Touati, D. Arch. Biochem. Biophy. 2000, 373, 1.

[3] (a) Perl, D. P.; Gajdusek, D. C.; Garruto, R. M.; Yanagihara, R. T.; Gibbs, C. J. Science 1982, 217, 1053.

(b) Crapper, D.; Krishnan, S.; Dalton, A. Science 1973, 180, 511.

[4] (a) Ta, C.; Reith, F.; Brugger, J.; Pring, A.; Lenehan, C. E. Environ.
Sci. Technol. 2014, 48, 5737.

(b) Armelao, L.; Bettinelli, M.; Rizzi, G. A.; Russo, U. J. Mater Chem. 1991, 1, 805.

(c) Peruchi, L. C.; Nunes, L. C.; Arantes de Carvalho, G. G.; Bueno Guerra, M. B.; de Almeida, E.; Rufini, I. A.; Santos, D., Jr.; Krug, F. J. Spectrochim. Acta B 2014, 100, 129.

[5] Mao, J.; Wang, L.; Dou, W.; Tang, X.; Yan, Y.; Liu, W. Org. Lett. 2007, 9, 4567 .

[6] (a) Azadbakht, R.; Vaisi, H.; Mohamadvand, H.; Khanabadi, J. Spectrochim. Acta A 2015, 145, 575.

(b) Lv, Y.; Zhu, L.; Liu, H.; Wu, Y.; Chen, Z.; Fu, H.; Tian, Z. Anal. Chim. Acta 2014, 839, 74.

(c) Zhan, S.; Xu, H.; Zhang, D.; Xia, B.; Zhan, X.; Wang, L.; Lv, J.; Zhou, P. Biosens. Bioelectron. 2015, 72, 95.

(d) Maiti, S.; Aydin, Z.; Zhang, Y.; Guo, M. Dalton Trans. 2015, 44, 8942 .

(e) Wang, M.; Wang, F.; Wang, Y.; Zhang, W.; Chen, X. Dyes Pigm. 2015, $120,307$.

(f) Prabhu, J.; Velmurugan, K.; Nandhakumar, R. J. Anal. Chem. 2015, 70, 943 .

(g) Antony, E. J.; Raj, M.; Paulpandi, R. Q.; Paulraj, M. S.; Enoch, I. V. M. V. J. Fluoresc. 2015, 25, 1031.

(h) Geng, T.-M.; Wu, D.-Y.; Huang, W.; Huang, R.-Y.; Wu, G.-H. J. Polym. Res. 2014, 21, 354.

(i) Xu, Z.; Baek, K.-H.; Kim, H. N.; Cui, J.; Qian, X.; Spring, D. R.; Shin, I.; Yoon, J. J. Am. Chem. Soc. 2009, 132, 601.

[7] (a) Chereddy, N. R.; Nagaraju, P.; Raju, M. V.; Krishnaswamy, V. R.; Korrapati, P. S.; Bangal, P. R.; Rao, V. J. Biosens. Bioelectron. 2015, 68, 749 .

(b) Hou, S. H.; Qu, Z. G.; Zhong, K. H.; Bian, Y. J.; Tang, L. J. Tetrahedron Lett. 2016, 57, 2616.

[8] Hou, S. H.; Qu, Z. G.; Zhong, K. H.; Bian, Y. J.; Tang, L. J. Chin. J. Org. Chem. 2016, 36, 768 (in Chinese).

(侯淑华, 曲忠国, 钟克利, 边延江, 汤立军, 有机化学, 2016, 36, 768.) 